\title{
Photonic crystal slow light devices fabricated by CMOS-compatible process
}

\author{
Toshihiko Baba ${ }^{\text {a) }}$, Hong C. Nguyen, Norihiro Ishikura, \\ Keijiro Suzuki, Mizuki Shinkawa, Ryo Hayakawa, \\ and Keisuke Kondo \\ Department of Electrical and Computer Engineering, \\ Yokohama National University, \\ 79-5 Tokiwadai, Hodogaya-ku, Yokohama 240-8501, Japan \\ a)baba@ynu.ac.jp
}

\begin{abstract}
We have studied wideband dispersion-free slow light in photonic crystal waveguides and other photonic nanostructures, which allows tunable delays in short optical pulses and the enhancement of light-matter interaction. This paper demonstrates the state-of-the-art devices fabricated by CMOS-compatible process, where compact devices of $200-400 \mu \mathrm{m}$ lengths are integrated with Si photonics components and controlled by DC and AC electronics. They are applied for dispersion controllers, optical correlators, DQPSK receivers, retiming of pulse train, two-photon absorption photo-diodes and Mach-Zehnder modulators.
\end{abstract}

Keywords: slow light, photonic crystal, silicon photonics, tunable delay, nonlinearity, photonic integration

Classification: Optoelectronics, Lasers and quantum electronics, Ultrafast optics, Silicon photonics, Planar lightwave circuits

\section{References}

[1] M. Notomi, K. Yamada, A. Shinya, J. Takahashi, C. Takahashi, and I. Yokohama, "Extremely large group velocity dispersion of line-defect waveguides in photonic crystal slabs," Phys. Rev. Lett., vol. 87, no. 25, pp. 253902-1-253902-4, Dec. 2001.

[2] T. Baba, "Slow light in photonic crystals," Nature Photon., vol. 2, no. 8, pp. 465-473, Aug. 2008.

[3] J. D. Joannopoulos, S. G. Johnson, J. N. Winn, and R. D. Meade, Photonic Crystals: Molding the Flow of Light, 2nd Ed., Princeton University Press, New York, 2008.

[4] T. Baba, A. Motegi, T. Iwai, N. Fukaya, Y. Watanabe, and A. Sakai, "Light propagation characteristics of straight single line defect optical waveguides in a photonic crystal slab fabricated into a silicon-on-insulator substrate," IEEE J. Quantum Electron., vol. 38, no. 7, pp. 743-752, July 2002.

[5] A. Sakai, G. Hara, and T. Baba, "Propagation characteristics of ultrahigh D optical waveguide on silicon-on-insulator substrate," Jpn. J. Appl. Phys., vol. 40, no. 4B, pp. L383-L385, April 2001. 
[6] E. Kuramochi, M. Notomi, S. Hughes, A. Shinya, T. Watanabe, and L. Ramunno, "Disorder-induced scattering loss of line-defect waveguides in photonic crystal slabs," Phys. Rev. B, vol. 72, no. 16, p. 161318, Oct. 2005.

[7] L. O'Faolain, S. A. Schulz, D. M. Beggs, T. P. White, M. Spasenovic, L. Kuipers, F. Morichetti, A. Melloni, S. Mazoyer, J. P. Hugonin, P. Lalanne, and T. F. Krauss, "Loss engineered slow light waveguides," Opt. Express, vol. 18, no. 26, pp. 27627-27638, Dec. 2010.

[8] D. Mori and T. Baba, "Dispersion-controlled optical group delay device by chirped photonic crystal waveguides," Appl. Phys. Lett., vol. 85, no. 7, pp. 1101-1103, June 2004.

[9] D. Mori and T. Baba, "Wideband and low dispersion slow light by chirped photonic crystal coupled waveguide," Opt. Express, vol. 13, no. 23, pp. 9398-9408, Nov. 2005.

[10] A. Sakai, I. Kato, D. Mori, and T. Baba, "Anomalous low group velocity and low dispersion in simple photonic crystal line defect waveguides," Tech. Dig. IEEE/LEOS Annual Meet., Puerto Rico, USA, no. ThQ5, Nov. 2004.

[11] A. Y. Petrov and M. Eich, "Zero dispersion at small group velocities in photonic crystal waveguides," Appl. Phys. Lett., vol. 85, no. 21, pp. 48664868, Sept. 2004.

[12] L. H. Frandsen, A. V. Lavrinenko, J. F. Pedersen, and P. I. Borel, "Photonic crystal waveguides with semi-slow light and tailored dispersion properties," Opt. Express, vol. 14, no. 20, pp. 9444-9450, Sept. 2006.

[13] S. Kubo, D. Mori, and T. Baba, "Low-group-velocity and low-dispersion slow light in photonic crystal waveguides," Opt. Lett., vol. 32, no. 20, pp. 2981-2983, Oct. 2007.

[14] J. Li, T. P. White, L. O'Faolain, A. G. Iglesias, and T. F. Krauss, "Systematic design of flat band slow light in photonic crystal waveguides," Opt. Express, vol. 16, no. 9, pp. 6227-6229, April 2008.

[15] Y. Hamachi, S. Kubo, and T. Baba, "Slow light with low dispersion and nonlinear enhancement in a lattice-shifted photonic crystal waveguide," Opt. Lett., vol. 34, no. 7, pp. 1072-1074, April 2009.

[16] M. Shinkawa, N. Ishikura, Y. Hama, K. Suzuki, and T. Baba, "Nonlinear enhancement in photonic crystal slow light waveguides fabricated using CMOS-compatible process," Opt. Express, vol. 19, no. 22, pp. 2220822218, Oct. 2011.

[17] F. Shinobu, Y. Arita, T. Tamanuki, N. Ishikura, and T. Baba, "Continuously tunable slow-light device consisting of heater-controlled silicon microring array," Opt. Express, vol. 19, no. 14, pp. 13557-13564, June 2011.

[18] J. Cardenas, M. A. Foster, N. Sherwood-Droz, C. B. Poitras, H. L. R. Lira, B. Zhang, A. L. Gaeta, J. B. Khurgin, P. Morton, and M. Lipson, "Wide-bandwidth continuously tunable optical delay line using silicon microring resonators," Opt. Express, vol. 18, no. 25, pp. 26525-26534, Dec. 2010.

[19] A. Melloni, F. Morichetti, C. Ferrari, and M. Martinelli, "Continuously tunable 1 byte delay in coupled-resonator optical waveguides," Opt. Lett., vol. 33, no. 20, pp. 2389-2391, Oct. 2008.

[20] F. Xia, L. Sekaric, and Y. Vlasov, "Ultracompact optical buffers on a silicon chip," Nature Photon., vol. 1, pp. 65-71, Jan. 2007.

[21] T. Tsuchizawa, K. Yamada, H. Fukuda, T. Watanabe, S. Uchiyama, and S. Itabashi, "Low-loss Si wire waveguides and their application to thermooptic switches," Jpn. J. Appl. Phys., vol. 45, no. 8B, pp. 6658- 
6662, Aug. 2006.

[22] N. Ishikura, R. Hayakawa, R. Hosoi, T. Tamanuki, M. Shinkawa, and T. Baba, "Photonic crystal tunable slow light device integrated with multiheaters," Appl. Phys. Lett., vol. 100, no. 22, p. 221110, May 2012.

[23] N. Ishikura, T. Baba, E. Kuramochi, and M. Notomi, "Large tunable fractional delay in slow light pulse and its application to fast optical correlator," Opt. Express, vol. 19, no. 24, pp. 24102-24108, Nov. 2011.

[24] K. Suzuki, H. C. Nguyen, T. Tamanuki, F. Shinobu, Y. Saito, Y. Sakai, and T. Baba, "Slow-light-based variable symbol-rate silicon photonics DQPSK receiver," Opt. Express, vol. 20, no. 4, pp. 4796-4804, Feb. 2012.

[25] K. Kondo, M. Shinkawa, Y. Hamachi, Y. Saito, Y. Arita, and T. Baba, "Ultrafast slow-light tuning beyond the carrier lifetime using photonic crystal waveguides," Phys. Rev. Lett., vol. 110, no. 5, p. 053902, Jan. 2013.

[26] M. F. Yanik and S. Fan, "Stopping light all optically," Phys. Rev. Lett., vol. 92, no. 8, p. 083901, Feb. 2004.

[27] Y. Saito and T. Baba, "Stopping of light by the dynamic tuning of photonic crystal slow light device," Opt. Express, vol. 18, no. 16, pp. 1714117153, July 2010.

[28] J. Upham, Y. Tanaka, Y. Kawamoto, Y. Sato, T. Nakamura, B. S. Song, T. Asano, and S. Noda, "Time-resolved catch and release of an optical pulse from a dynamic photonic crystal nanocavity," Opt. Express, vol. 19, no. 23, pp. 23377-23385, Nov. 2011.

[29] R. Hayakawa, N. Ishikura, and T. Baba, "Two-photon-absorption photodiodes in Si photonic-crystal slow-light waveguides," Appl. Phys. Lett., vol. 102, no. 3, p. 031114, Jan. 2013.

[30] T. K. Liang, H. K. Tsang, I. E. Day, J. Drake, A. P. Knights, and M. Asghari, "Silicon waveguide two-photon absorption detector at $1.5 \mu \mathrm{m}$ wavelength for autocorrelation measurements," Appl. Phys. Lett., vol. 81, no. 7, pp. 1323-1325, June 2002.

[31] T. Tanabe, H. Sumikura, H. Taniyama, A. Shinya, and M. Notomi, "Allsilicon sub-Gb/s telecom detector with low dark current and high quantum efficiency on chip," Appl. Phys. Lett., vol. 96, no. 10, p. 101103, March 2010.

[32] H. Chen and A. W. Poon, "Two-photon absorption photocurrent in p-i-n diode embedded silicon microdisk resonators," Appl. Phys. Lett., vol. 96, no. 19, p. 191106, May 2010.

[33] H. C. Nguyen, Y. Sakai, M. Shinkawa, N. Ishikura, and T. Baba, "Photonic crystal silicon optical modulators: carrier-injection and depletion at $10 \mathrm{~Gb} / \mathrm{s}, "$ IEEE J. Quantum Electron., vol. 48, no. 2, pp. 210-220, Jan. 2012.

[34] H. C. Nguyen, S. Hashimoto, M. Shinkawa, and T. Baba, "Compact and fast photonic crystal silicon optical modulators," Opt. Express, vol. 20, no. 20, pp. 22465-22474, Sept. 2012.

[35] G. T. Reed, G. Mashanovich, F. Y. Gardes, and D. J. Thomson, "Silicon optical modulators," Nature Photon., vol. 4, no. 8, pp. 518-526, July 2010.

\section{Introduction}

Slow light is light with a group velocity $v_{\mathrm{g}}$ much smaller than that in vacuum c. The group index $n_{\mathrm{g}}$ denoting the slowdown factor of optical pulses is 
defined by $n_{\mathrm{g}} \equiv c / v_{\mathrm{g}}$, and is given by

$$
n_{\mathrm{g}}=c(d k / d \omega)=n+\omega(\Delta n / \Delta \omega) \approx \Delta n /(\Delta \omega / \omega) \text { when } \quad n_{\mathrm{g}} \gg n
$$

where $k$ is the wave number, $\omega$ is the frequency and $n$ is the material or modal index. Eq. (1) indicates that slow light is generated by the large first order dispersion in some anomalous media and photonic nanostructures. However, since $\Delta n$ cannot be higher than 1 in any cases, Eq. (1) also suggests that a large $n_{\mathrm{g}}$ is obtainable in a particularly small $\Delta \omega$; in other words, $n_{\mathrm{g}}$ and $\Delta \omega$ are trade-off. In addition, the large first order dispersion is often accompanied by large higher-order dispersions [1], which expand and deform the pulses severely and hampers the wide range of applications of slow light.

Based on such backgrounds, we have studied wideband dispersion-free slow light in photonic nanostructures and particularly photonic crystal (PC) waveguides [2]. Nanostructures consisting of high-index-contrast media achieve a large $\Delta n$ over 0.1 and allow wider design flexibility for suppressing higher order dispersions. In this paper, we give an overview of nanostructure-based slow light and its applications. In the next section, we first summarize expected effects and potential applications. In Section 3, we discuss dispersionfree slow light and some structures. In Section 4, we describe the device fabrication using Si CMOS- compatible process, one of the recent progress toward large-scale integrations of slow light devices. In Sections 5, we show some applications demonstrated to date. Note that we omit the fundamentals of PCs and standard PC line-defect waveguides. For further studies on these matters, please refer to $[3,4]$.

\section{Expectations and applications}

The $n_{\mathrm{g}}-\Delta \omega$ constraint is essential for any slow light, provided that the optical spectrum is conserved during the propagation. Therefore, one should know the maximum $n_{\mathrm{g}}$ expected for one's target bandwidth. Let us assume common values $n=3$ and $\Delta n=0.2$ in photonic nanostructures at wavelengths $\lambda \sim 1.55 \mu \mathrm{m}$. If slow light is applied to 100-Gbps non-return-to-zero (NRZ) signals, for example, the relative bandwidth is $\Delta \omega / \omega=0.00052$ and $n_{\mathrm{g}}$ derived from Eq. (1) is $\sim 380$ (its enhancement factor $\xi$ against $n_{\mathrm{g}} \approx 4.2$ in $\mathrm{Si}$ wire waveguides [5] is 90). If it is applied to Gaussian pulses with a full-width at half maximum (FWHM) of $1 \mathrm{ps}, \Delta \omega / \omega=0.0023$ and $n_{\mathrm{g}}=90$ $(\xi=21)$. If the full C-band operation at $\lambda=1.530-1.565 \mu \mathrm{m}$ is necessary, $\Delta \omega / \omega=0.023$ and $n_{\mathrm{g}}=12(\xi=2.9)$. In earlier studies, huge $n_{\mathrm{g}}$ over $10^{4}$ were focused and investigated, although their bandwidths were very narrow. As compared to them, the above wideband $n_{\mathrm{g}}$ appears to be small, but still the above $n_{\mathrm{g}}$ with sufficient bandwidths are advantageous in many applications. From more practical points of view, $n_{\mathrm{g}}>100$ is difficult to use because it causes a large loss. As slow light enhances the interaction with the structure, the scattering loss due to the structural disordering is expected to increase linearly with $n_{\mathrm{g}}$. It has been indicated, however, that $n_{\mathrm{g}}>40$ accelerates the scattering into backward slow modes, resulting in 
the $n_{\mathrm{g}}{ }^{2}$-dependent loss $[6,7]$. The threshold $n_{\mathrm{g}}$ between the $n_{\mathrm{g}}$ and $n_{\mathrm{g}}{ }^{2}$ dependences can be increased by reducing the disordering, while at present, $n_{\mathrm{g}}<40(\xi<10)$ is a reasonable value for avoiding the severer loss.

Figure 1 summarizes possible applications of slow light. They are categorized into those using tunable delays and enhanced light-matter interaction. Tunable delays have been anticipated to achieve optical buffer memories in next era photonic networks. However, they are still challenging targets as they require a long buffering time of over $1 \mu \mathrm{s}$, which corresponds to a propagation length of $7.5 \mathrm{~m}$ with $n_{\mathrm{g}}=40$. Such long propagation cannot be obtained with a moderate loss. There are other more realistic applications of tunable delays. Retiming and multi-/demulti-plexing (MUX/DEMUX) of pulses do not require long delays but a tuning response time shorter than pulse width. If the group velocity dispersion (GVD) is controllable, it can be used for dispersion compensation. Even though the tuning response is slow, the tunable delay makes the demodulation rate of differential quad-phase shift keying (DQPSK) receivers to be variable. Arbitrary delay tuning in a multibranching circuit can conduct digital signal processing of short pulses such as finite impulse response (FIR) filters. Those in arrayed waveguides allows efficient phase array antennas at micro-/millimeter-wave frequencies. They will also miniaturize and simplify the pump-probe and correlation measurement systems, which are currently using mechanically tunable delay lines. On the other hand, the enhanced light-matter interaction occurs due to the elongated duration of light propagation as well as enhanced peak intensities of pulses. They will increase various optical nonlinearities such as two-photon absorption (TPA), self-phase modulation (SPM) and four-wave mixing (FWM), and improve the efficiency of phase modulation and optical amplification.

\section{Tunable delay in DC slow light}

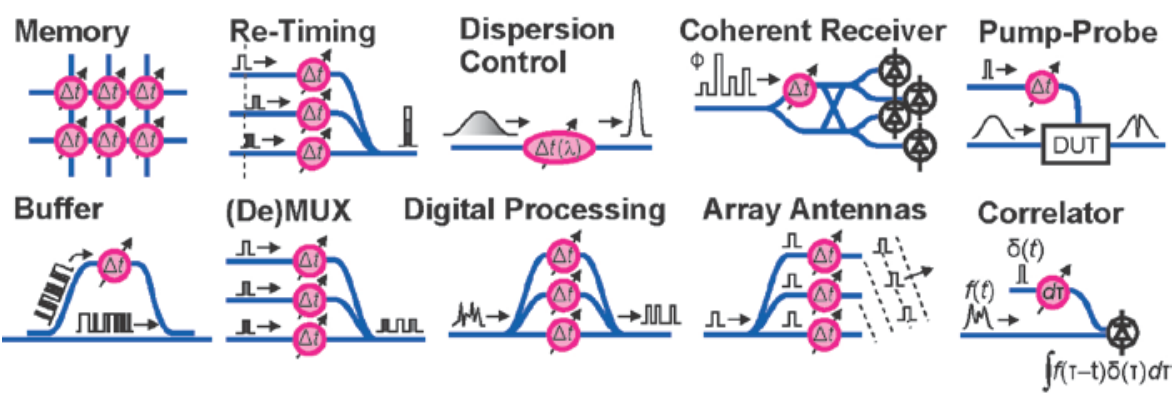

Enhancement of light-matter interaction with LD slow light
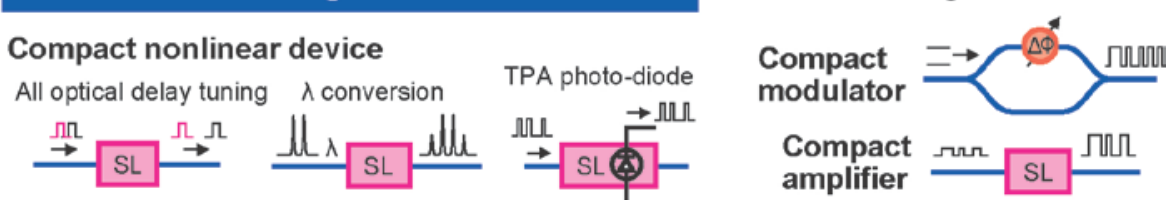

Fig. 1. Possible applications of slow light. 


\section{Dispersion-engineered slow light}

The suppression of higher order dispersions is important for most applications of slow light. There are two choices for this, i.e. dispersion-compensated $(D C)$ slow light $[8,9]$ and originally low-dispersion $(L D)$ slow light $[10,11$, 12, 13, 14], as shown in Fig. 2. The DC slow light is generated by a chirped structure, in which some structural parameters are changed gradually so that the photonic band is shifted smoothly. Let us consider a flat band $\left((d k / d \omega)^{-1}\right.$ is zero) located on an inflection point (DC band) and shifted linearly in the chirped structure. Then, the input optical pulse is first expanded by the first dispersion. Each frequency component reaches the slow light condition of the flat band at different position. Finally, the initial pulse shape is recovered by the second dispersion. In this operation, the chirp range determines the bandwidth of slow light and hence $n_{\mathrm{g}}$ on the basis of Eq. (1). This means that the delay is enhanced or reduced by decreasing or increasing the chirp range, respectively. As the internal pulse intensity is suppressed by the first dispersion, optical nonlinearities are not enhanced as those in LD slow light. The LD slow light is generated by a straight band with a small slope (LD band). Pulses are compressed in space and their peak intensities are enhanced by the ratio $\xi$ and propagates without changing its waveform, resulting in high nonlinearities. It is not suitable for tunable delays as the $n_{\mathrm{g}}$ is fixed by the slope of the straight band.

An example photonic nanostructure is the lattice-shifted PC waveguide (LSPCW, Fig. 3(a)) [15, 16]. In a standard photonic crystal waveguide in the PC slab consisting of hole arrays and a line defect, slow light only occurs near the photonic band edge. In the LSPCW, the third rows of holes from the line defect are shifted along the waveguide. Then it turns out to exhibit a flat band at a high frequency and a straight band at lower frequencies simultaneously (Fig. 3(b)). Thus it can be used for generating DC and LD slow light at different frequencies. Similar operation is obtainable in

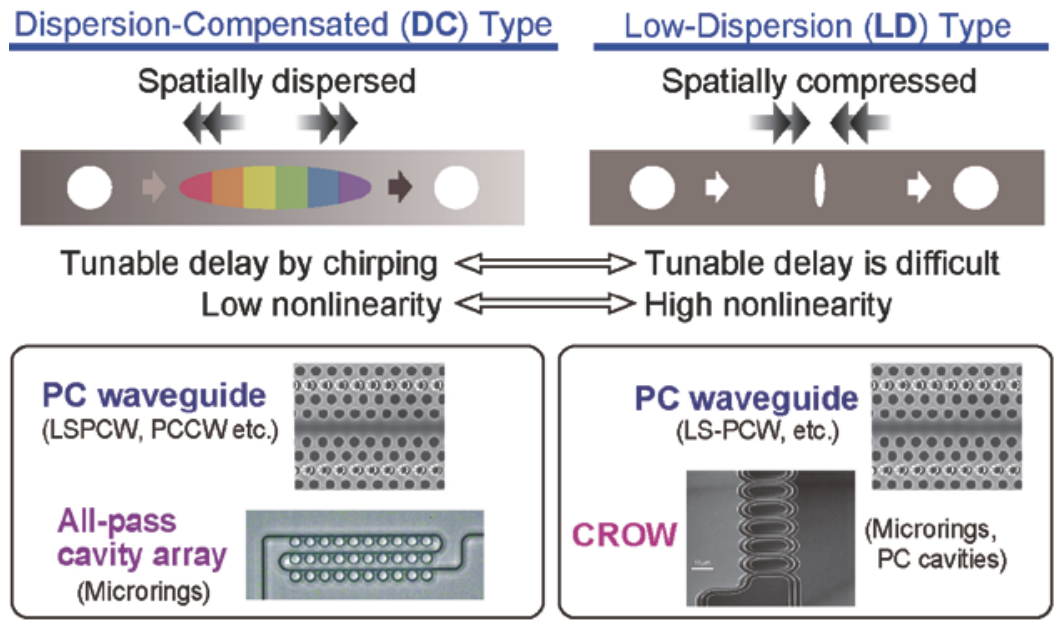

Fig. 2. Schematic of two-types of dispersion-engineered slow light pulse (upper panel) and photonic nanostructures which generate them (lower panel). 


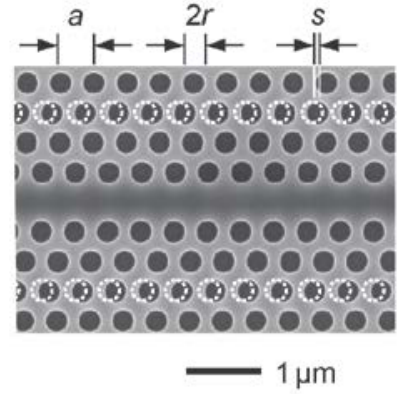

(a)

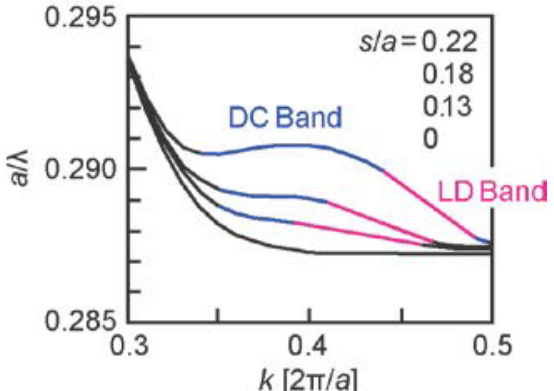

(b)

Fig. 3. LSPCW. (a) Top view of fabricated device and parameters. (b) Photonic bands for different lattice shift $s$.

many other PC waveguides, in which other holes are shifted in other directions and/or size-changed. However, they usually require the simultaneous optimization of multiple parameters and sometimes smaller holes or smaller inter-hole spacing. In the LSPCW, on the other hand, $n_{\mathrm{g}}$ and target wavelengths are controlled independently by the lattice shift and unchanged hole diameter, respectively, allowing large tolerance in the fabrication.

DC and LD slow light are also obtainable in cavities. For example, microrings individually coupled to a bus waveguide like all-pass filters (Fig. 2) can be a DC structure $[17,18,19]$. Here, light propagating in the bus waveguide is coupled with resonant rings. When the coupling is set to be so strong as to dominate the photon lifetime, coupled light is delayed by the lifetime. If rings have different resonant wavelengths, different wavelength light are delayed at different rings, similarly to the case of the chirped LSPCW. When the number of rings are limited, their in-phase and out-of-phase conditions form a complex transmission spectrum. But when the number is large, these conditions are averaged and the transmission is flattened. Thus, wideband slow light and tunable delays are available by controlling the resonant wavelengths by e.g. heating rings. On the other hand, the coupled resonator optical waveguide (CROW) consisting of series-coupled rings (Fig. 2) can be a LD structure [20]. It forms a pseudo straight band allowing the pulse propagation. It enhances the nonlinearities, while tunable delays are difficult as it requires the external control of the coupling strength between rings.

\section{Fabrication by CMOS-compatible process}

Si and related materials have often been used for PCs. In these years, CMOScompatible Si photonics foundry services have a big impact on their research and development. Common foundries use $8^{\prime \prime}$ or $12^{\prime \prime}$ silicon-on- insulator (SOI) wafers and stepper lithography at $\lambda=248$ or $193 \mathrm{~nm}$ whose resolution is 180 or $130 \mathrm{~nm}$, respectively. Therefore, hole diameters over $200 \mathrm{~nm}$ are acceptable in the process, and $\mathrm{SiO}_{2}$-cladded $\mathrm{PC}$ slabs are straightforward to fabricate. Even air-bridge PC slabs often employed in PC devices for the strong optical confinement, wide working spectrum and large $n_{\mathrm{g}}$ of slow light have become 


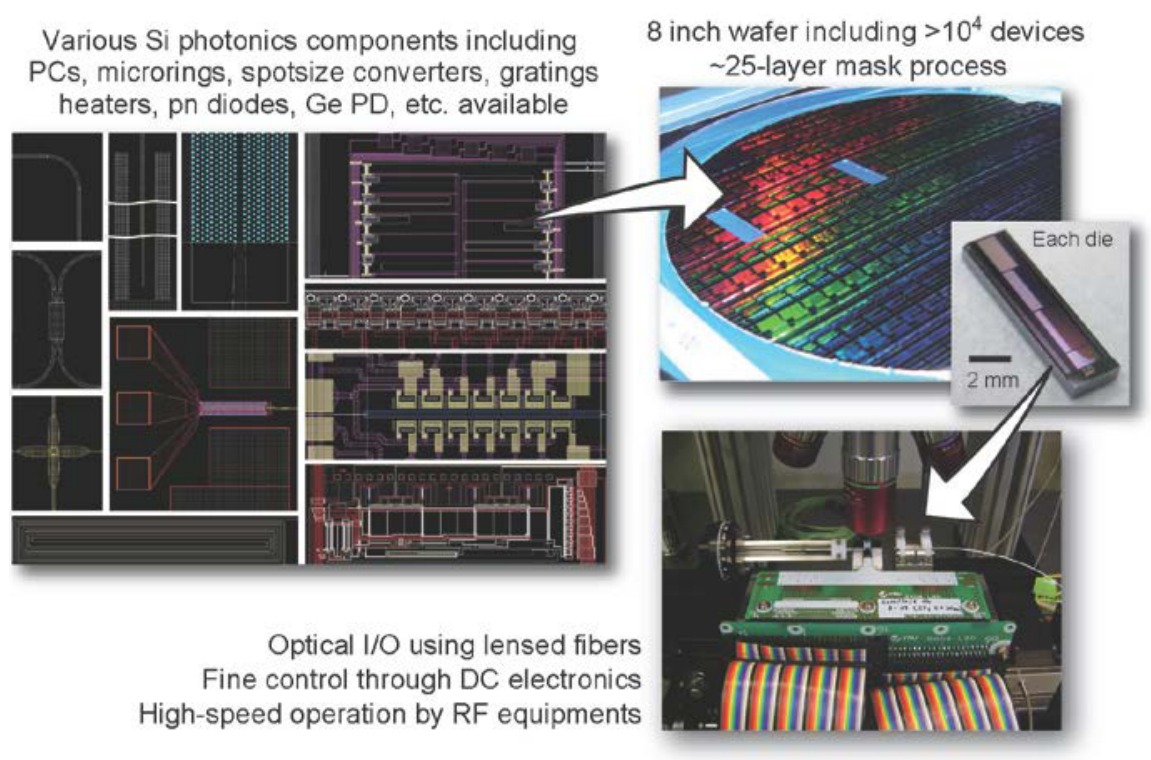

Fig. 4. Research and development using foundry service. GDS-II data of devices (left), wafer and die (right upper) and measurement (right lower).

available in the wafer scale although the process is complicated [16]. We can now integrate PCs with Si photonics components such as wire waveguides, microrings, spotsize converters (SSCs), gratings, heaters, pn diodes and epitaxial Ge photo-diodes (PDs) through more than 20 layer mask process, as shown in Fig. 4. We usually dice the wafer into dies for the end-fire optical coupling and mount each die on a printed circuit board to apply a DC bias to each component. For the optical coupling, light from lensed fiber is coupled to the SSC with a standard coupling loss of $2.5 \mathrm{~dB}$. The loss is determined by the sharpness of the inverse taper buried in the SSC and can be reduced to $<0.5 \mathrm{~dB}$ if we can use state-of-the-art high-resolution lithography [21].

In the process with $\lambda=248 \mathrm{~nm}$ lithography, the propagation loss of $\mathrm{Si}$ wires and PC waveguides at $n_{\mathrm{g}}<6$ are typically 3 and $10 \mathrm{~dB} / \mathrm{cm}$, respectively. The coupling loss between them is less than $1 \mathrm{~dB}$. Therefore, we can launch light into PC waveguides with a $3-4 \mathrm{~dB}$ loss. The loss increases in proportion to $n_{\mathrm{g}}$ at $n_{\mathrm{g}}<30$. Therefore, the loss due to slow light can be within $1 \mathrm{~dB}$ when we limit $n_{\mathrm{g}} \leq 30$, the device length $L \leq 400 \mu \mathrm{m}$, and delays up to $40 \mathrm{ps}$. These values will be enhanced by employing higher resolution lithography.

\section{Demonstrations}

\subsection{Tunable delays and dispersion [22]}

Figure 5 (a) shows the 320- $\mu$ m-long air-bridge LSPCW integrated with Si wire access waveguides and seven pairs of side heaters. Even with no chirping, some structural disordering fluctuates the photonic band. Therefore, slight heating some heaters compensates the disordering, uniforms the flat band frequency, and forms a sharp delay peak. Further heating disorders the 

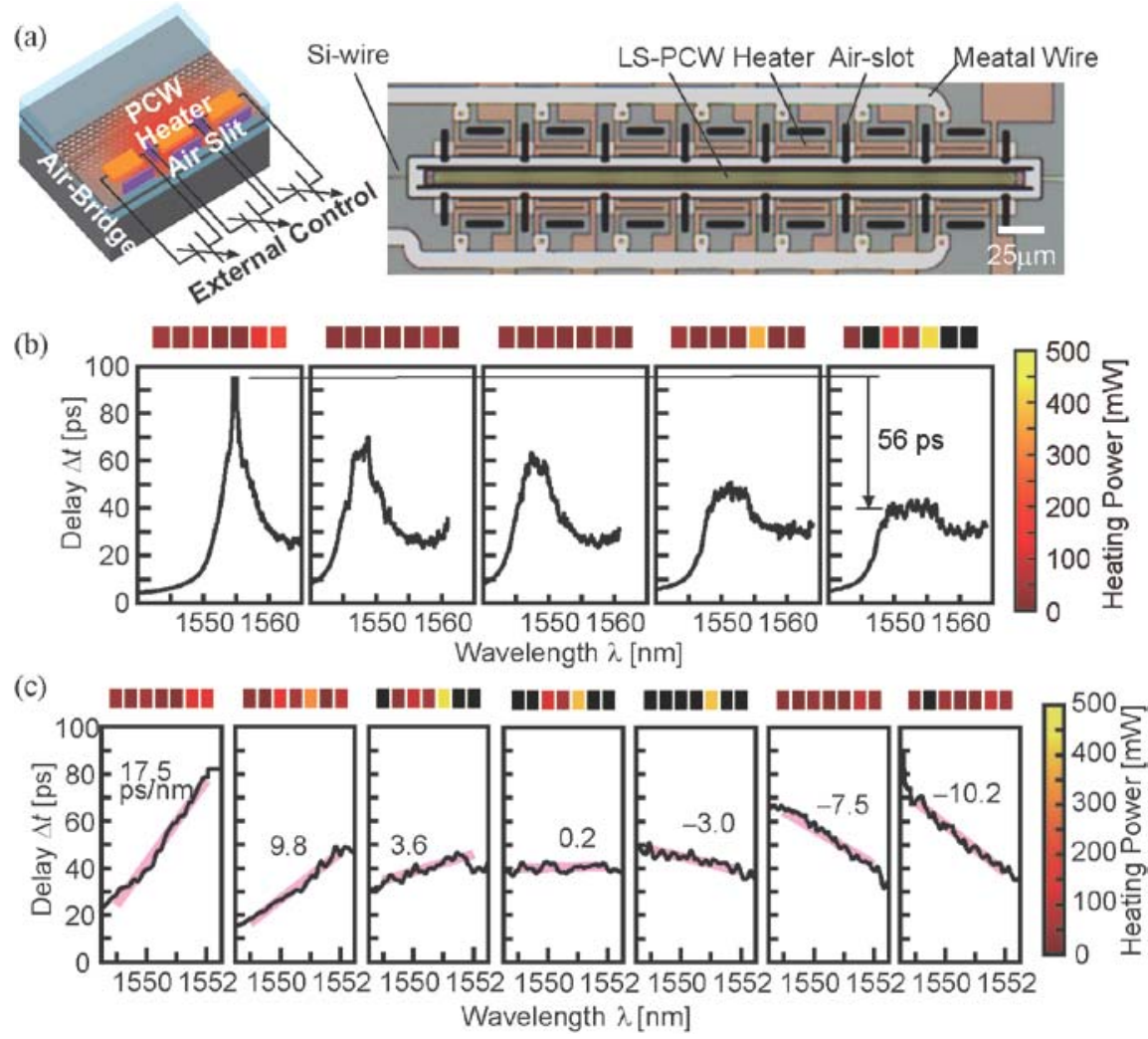

Fig. 5. LSPCW integrated with multi-heaters.

(a) Schematic and top view of fabricated device.

(b) Tunable delays. (c) Tunable GVD. In (b) and

(c), color squares depict heating powers at seven pairs of heaters.

uniformity again and broadens the delay spectrum, maintaining its flat top. In Fig. 5 (b), the maximum tuning range of the delay is $56 \mathrm{ps}$. Such tunable delays with a relatively slow tuning speed can be used as a delay scanner in optical correlators. It has been demonstrated not with integrated heaters but using local laser irradiation [23].

For Fig. 5 (b), the device is linearly chirped locally. If the local chirp has a quadratic distribution, the GVD is changed continuously, as shown in Fig. 5 (c). Positive and negative quadric chirpings formed by the heating give the negative and positive GVD, respectively. The tunable range from -10.2 to $+17.5 \mathrm{ps} / \mathrm{nm}$ for $320 \mu \mathrm{m}$ length corresponds to huge GVD coefficients from -31.9 to $54.7 \mu \mathrm{s} / \mathrm{nm} / \mathrm{km}$. This tunable dispersion has been applied to compressing pre-chirped optical pulses from 5.9 ps to $2.3 \mathrm{ps}$.

\subsection{Symbol-rate variable DQPSK receiver [24]}

DQPSK is a coherent modulation format that doubles the transmission speed even without local light sources in receivers. Pre-coded signals are demodulated by taking the difference between successive bits. For this, the input signal is first divided into two arms, and one of them is delayed by one signal bit. Then they are interfered in the $90^{\circ}$ hybrid and detected by a pair of balanced PDs. Figure 6 shows that all the components were integrated by the 


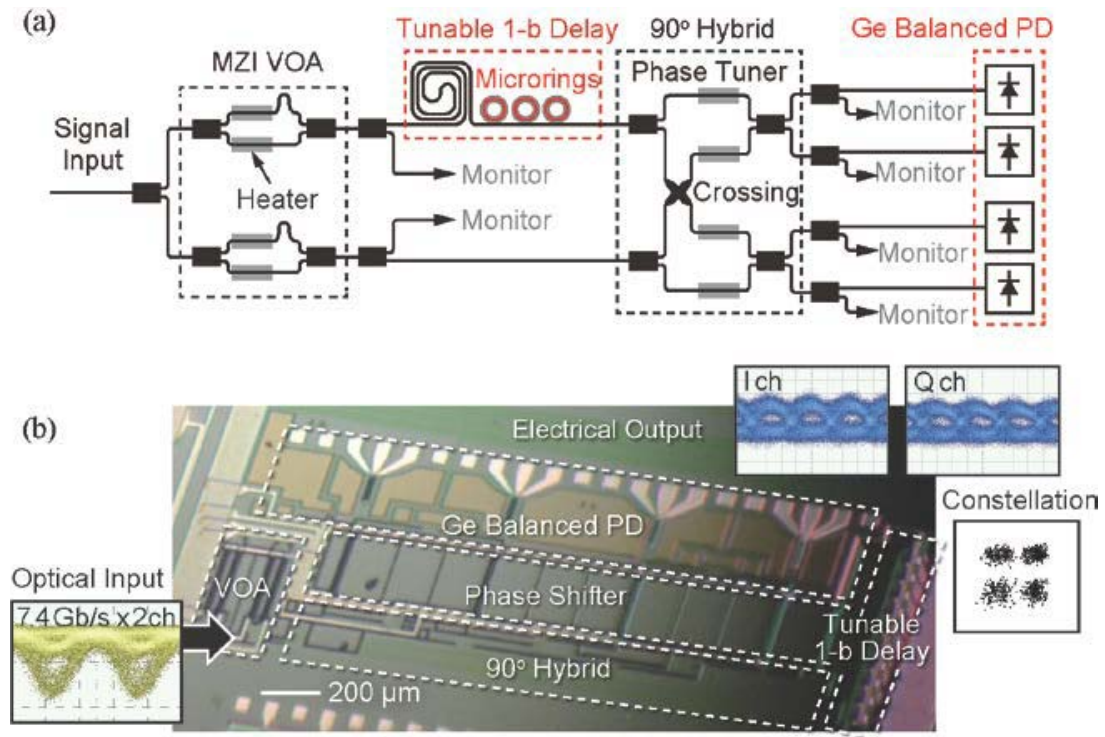

Fig. 6. Symbol-rate-variable DQPSK receiver.

(a) Schematic diagram. (b) Fabricated device and input optical/output electrical signals at 7.4 Gbaud $\times 2 \mathrm{ch}$.

CMOS-compatible process into a footprint of $2.5 \times 0.8 \mathrm{~mm}^{2}$. Optical intensities of the two arms are balanced by Mach-Zehnder interferometer (MZI) variable optical attenuators (VOAs). The interference condition in the $90^{\circ}$ hybrid is optimized by heater phase shifters. Epitaxial Ge PDs are used as the balanced PDs. The key component in this device is the tunable one-bit delay line. Usually, DQPSK receives are used in the narrow range of symbol rates determined by the one-bit delay line. The range of the symbol rates are expanded by a tunable delay line. Here, the heater-controlled all-pass microrings are used for tuning the delay from 100 to $150 \mathrm{ps.} \mathrm{Adding} \mathrm{a} \mathrm{fixed}$ delay line, the one-bit delay is obtained for symbol rates from 7.4-9.0 Gbaud. Eye openings for I and Q channels as well as separated constellation patterns were observed at bitrates of $14.8-18.0 \mathrm{Gbps}$

\subsection{All optical ultrafast delay tuning [25]}

The delay tuning shown above are driven by the heating whose response time was measured to be $19 \mu \mathrm{s}$ for the air-bridge PC slab [23]. It is too slow for retiming and MUX/DEMUX of short pulses. For the fast delay tuning, we use the LD slow light as the control pulse (Fig. 7 (a) and (b)). A high-intensity LD pulse generates TPA in Si, inducing carrier plasma effects and the blue-shift of the photonic band. When the DC signal pulse is incident on $200-\mu \mathrm{m}$-long LSPCW later than the LD pulse, the delay of the DC pulse is increased by the blue-shifted photonic band in the case of Fig. 7. Here, the response of the delay tuning is limited by the carrier lifetime of $120 \mathrm{ps.} \mathrm{When} \mathrm{the} \mathrm{DC}$ pulse is incident earlier, the LD pulse with a slightly higher $v_{\mathrm{g}}$ overtakes the DC pulse. At this moment, the DC pulse suffers the dynamic tuning of the LD pulse $[26,27,28]$ and the delay is reduced by $10 \mathrm{ps}$ at maximum. Here, 


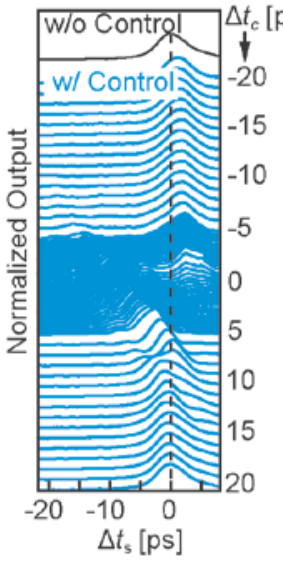

(a)

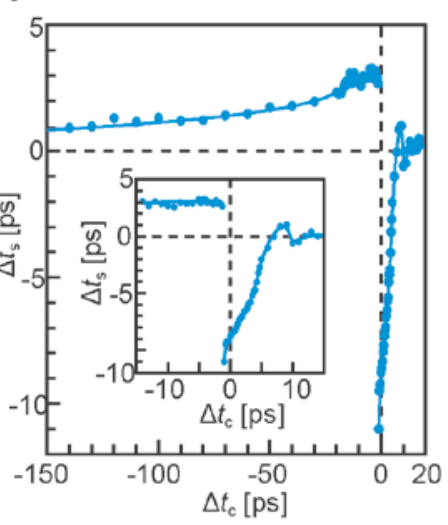

(b)

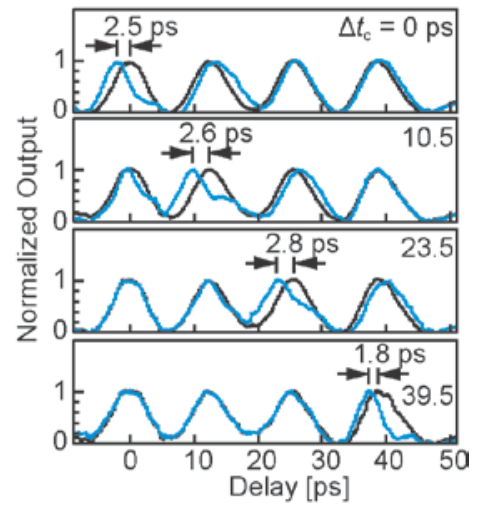

(c)

Fig. 7. Ultrafast delay tuning in LSPCW. $\Delta t_{\mathrm{s}}$ is the delay of DC slow light pulse. $\Delta t_{\mathrm{c}}$ is the incident timing of LD slow light pulse against the DC pulse.

(a) Cross-correlation waveform of DC pulse. (b) $\Delta t_{\mathrm{s}}$ versus $\Delta t_{\mathrm{c}}$ characteristics. (c) Selective delay tuning of one target pulse in high-speed pulse train.

the response is not limited by the carrier lifetime but the overlap duration of the two pulses and can be shorter than $10 \mathrm{ps.} \mathrm{This} \mathrm{allows} \mathrm{the} \mathrm{selective} \mathrm{delay}$ tuning of one target pulse in high-speed pulse train (Fig. 7 (c)). Here, the pulse train of $12 \mathrm{ps}$ interval was produced in a Si wire circuit and incident on the LSPCW together with one LD pulse. The delay of the pulse overlapping with the LD pulse in the LSPCW changes its delay. It was suggested that the retiming DC signal pulses is possible by inserting LD pulses as a clock and setting their timing appropriately.

\subsection{TPA-enhanced PD [29]}

The TPA occurs in a long Si waveguides when the optical intensity is high. This has been applied for detecting long wavelength light in Si [30]. To reduce the waveguide length and improve the responsivity, cavity-based TPA-PDs were fabricated, while their bandwidths were too narrow to detect short pulses [31, 32]. LD slow light is more suitable for high responsivity operation with a sufficient bandwidth for short pulses. We fabricated pn-diode-loaded LSPCW and observed the photocurrent (Fig. 8 (a), (b)). At a $-3 \mathrm{~V}$ bias, the dark current was as low as $40 \mathrm{pA}$. The photocurrent was observed for average optical powers from sub- $\mu \mathrm{W}$ to $1 \mathrm{~mW}$ and corresponding pulse peak powers from sub- $10 \mathrm{~mW}$ to sub-10 W. Except around upper and lower limits, the responsivity is proportional to the square of the incident power, indicating the TPA action. Higher $n_{\mathrm{g}}$ improves the responsivity, and the theory including the $n_{\mathrm{g}}{ }^{2}$-dependent TPA well explains the experiment.

The TPA-PD can be used as a detector in optical correlators. Figure 8 (c) shows auto-correlation traces of pulses, which are in good agreement with those obtained in commercial correlators. The TPA-PD is also applicable for

(C) IEICE 2013
DOI: $10.1587 /$ elex.10.20132002 Received March 18, 2013 Accepted April 01, 2013 Published May 25, 2013 


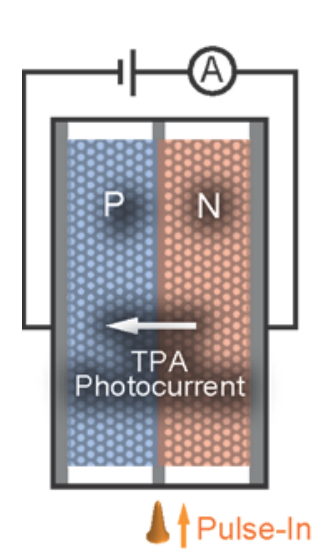

(a)

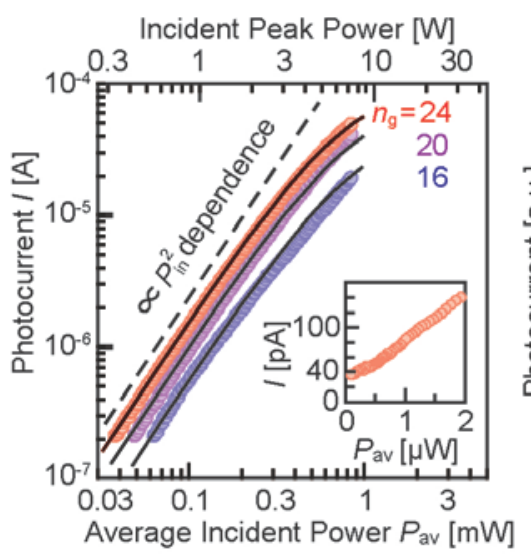

(b)

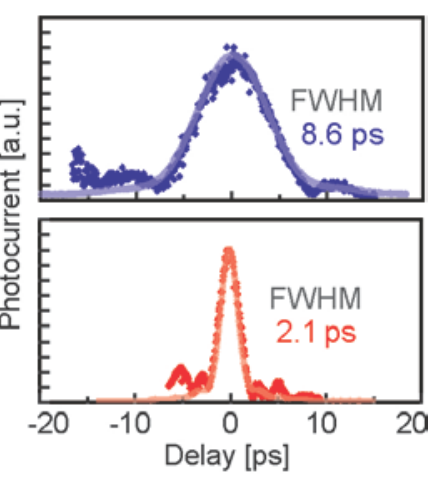

(c)

Fig. 8. Si LSPCW TPA-PD at $\lambda \sim 1.55 \mathrm{~mm}$.

(a) Schematic of the device. (b) Experimental (circle) and theoretical (lines) response characteristics. (c) Auto-correlation traces of pulses observed with the PD (dots) and commercial correlator (solid line).

detecting the dispersion in pulses. In linear PDs, the photocurrent is proportional to the time-integral of the pulse, so independent of the dispersion. On the other hand, the photocurrent from the TPA-PD is inversely proportional to the pulse width.

\subsection{Compact MZI modulator [33, 34]}

Optical modulators are one of the key components in Si-photonics-based optical interconnects, and three kinds of modulators have been developed actively in these ten years, i.e. MZI-type, resonator-type and electro-absorption (EA) type [35]. The MZI-type has a wide working spectrum but its size is of millimeter order. The resonator-type is much smaller while its working spectrum is narrower than $1 \mathrm{~nm}$. The EA-type requires complicated processes for epitaxy and fabricating waveguides and still has small advantage over the other. Slow light is effective for limiting the working spectrum moderately and reducing the size of the MZI-type. In Si MZI modulators, phase shifters consisting of $\mathrm{p}(\mathrm{i}) \mathrm{n}$-diodes change the phase of guided waves by $\Delta \phi=\Delta k L$ through the carrier plasma dispersion. In the slow light regime, $\Delta k$ is enhanced by $\xi$, meaning that $\xi$-fold shorter device is possible. For the $\mathrm{SiO}_{2}$-clad LSPCW, we can expect $n_{\mathrm{g}}=20-25(\xi \sim 6)$ with $\Delta \lambda=15 \mathrm{~nm}$ at $\lambda \sim 1.55 \mu \mathrm{m}$ as practical and realistic values. Conventional 1-mm-long phase shifters of rib waveguides can be shorter than $200 \mu \mathrm{m}$ with the LSPCW. Actually, we may be able to expect further size reduction in high-speed modulators because such short lengths $L \sim 100 \mu \mathrm{m}$ allow the modulators to operate as lumped parameter devices at data rates of $10 \mathrm{Gbps}$ order.

Figure 9 demonstrates such Si MZI LSPCW at 10 and 40 Gbps. We fabricated two structures; with phase shifters in both arms (dual-type) and with phase shifter in one arm (single). The eye opening was observed at 
10 Gbps in the $50-\mu \mathrm{m}$-long dual device over a wide spectrum of $\Delta \lambda=12.5 \mathrm{~nm}$, and at $40 \mathrm{Gbps}$ in the $90-\mu \mathrm{m}$-long single device. Even though the error-free operation is obtained in slightly longer devices, such markedly short devices strongly demonstrate the advantage of slow light effect on the practical device level.

(a)

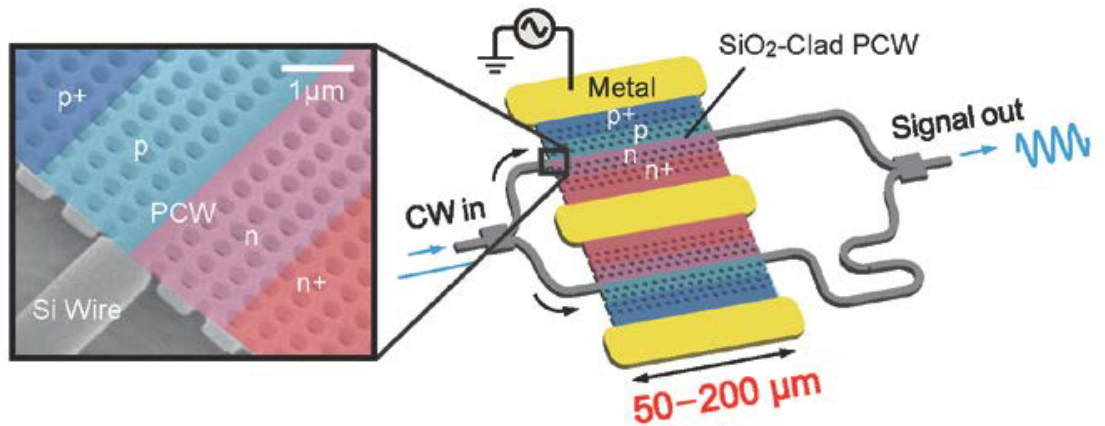

(b)

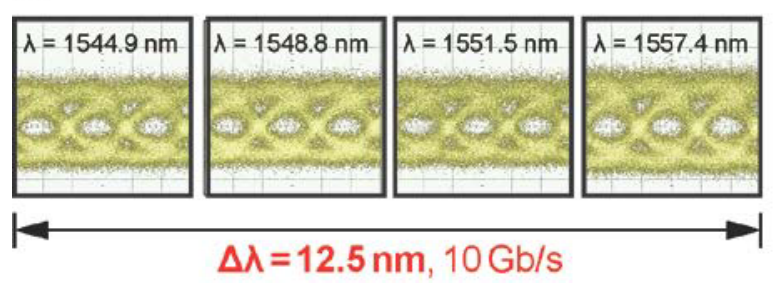

(c)

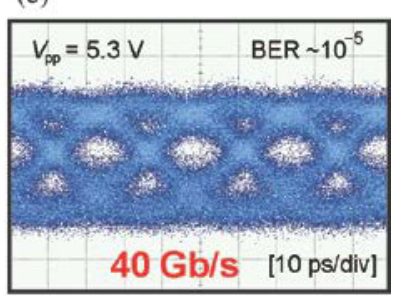

Fig. 9. Si MZI LSPCW modulators.

(a) Schematic of dual-type modulator and scanning electron micrograph of fabricated LSPCW with $\mathrm{p}+, \mathrm{p}, \mathrm{n}, \mathrm{n}+$ regions colored. (b) Eye patterns at $10 \mathrm{Gbps}$ with dual $50-\mu \mathrm{m}$-long LSPCW phase shifters. (c) Eye pattern at 40 Gbps with single $90-\mu \mathrm{m}$-long PC phase shifter.

\section{Conclusions}

Slow light was studied formerly as attractive physics that achieves huge $n_{\mathrm{g}}$. However, we now realize that balancing $n_{\mathrm{g}}$, bandwidth and loss is essentially important, and that moderate $n_{\mathrm{g}} \leq 40$ already gives rise to significant advantages in various applications. With the rapid progress of Si CMOScompatible process, some devices such as MZI modulators are getting close to the practical use.

\section{Acknowledgments}

This work was supported by the FIRST Program of JSPS and Future Pioneering Projects of METI. 

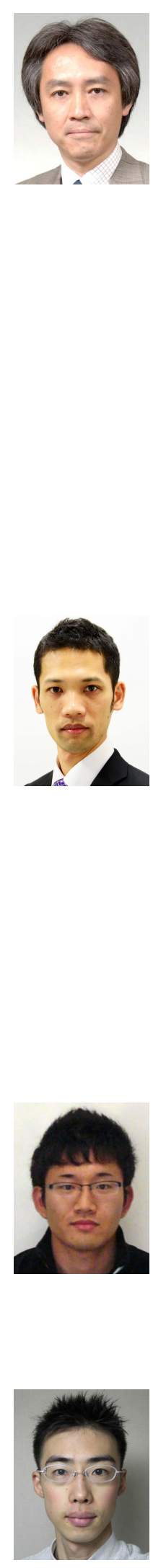

(c) IEICE 2013

DOI: $10.1587 /$ elex.10.20132002

Received March 18, 2013

Accepted April 01, 2013

Published May 25, 2013

\section{Toshihiko Baba}

received the B. E., M. E., and Ph.D. degrees from the Division of Electrical and Computer Engineering, Yokohama National University (YNU), Japan, in 1985, 1987, and 1990, respectively. From 1991 - 1993, he joined Tokyo Institute of Technology as a research associate. He became an associate professor and full professor of YNU in 1994 and 2005, respectively. In these 20 years, he has studied ARROW waveguides, long-wavelength VCSELs, photonic crystals (PCs), and Si photonics. He has discussed the spontaneous emission control in microcavities, and demonstrated PC nanolasers, slow light waveguides, PC LEDs, compact Si AWG demultiplexers, high-speed Si Mach-Zehnder modulators, and so on. He is the author and co-author of more than 150 papers with more than 7300 citations.

Dr. Baba is a member of the Institute of Electronics, Information and Communication Engineers, the Japan Society of Applied Physics (JSAP), IEEE Photonics Society and the Optical Society of America (OSA). He received 14 academic awards including JSPS Award in 2005, LEOS Distinguished Lecturer Award in 2006/2007, IEICE Electronics Society Award in 2011, and Ichimura Academic Award in 2012.

\section{Hong C. Nguyen}

received a B.Sc (Honours) and Ph.D degrees in physics at the Centre for Ultrahigh-bandwidth Devices for Optical Systems (CUDOS), School of Physics, University of Sydney, Australia, in 2003 and 2008 respectively. He was a research associate at the Department of Electrical and Computer Eng., YNU until 2013. He is currently Global Sales \& Marketing at Alnair Labs Corporation, Japan.

His research interests include nonlinear optics and Si photonics. He is a member of OSA and JSAP, and was president of the OSA Student Chapter at the University of Sydney in 2006/2007. He was a finalist for the OSA/New Focus Bookham Student Prize in 2007, and received the Optium Student Prize at OECC/ACOFT in 2008. He also received the Young Scientist Oral Presentation Award at JSAP Annual Spring Meeting and the Best Post-Deadline Paper at Group IV Photonics in 2012.

\section{Norihiro Ishikura}

received the B. E. and M. E. degrees from the Department of Electrical and Computer Engineering, YNU, in 2009 and 2011, respectively. He is currently working towards the Ph.D. degree in Si photonics slow light devices at the same university, receiving the Research Fellowship for Young Scientists from Japan Society for the Promotion of Science (JSPS). He is a member of JSAP. He received the Paper Award at Micro-Optic Conference 2011.

\section{Keijiro Suzuki}

received the B. E. and M. E. degrees from the Department of Electrical and Electronic Engineering, Shizuoka University, Japan, in 2004 and 2006, respectively. After spending two years at Sumitomo Osaka Cement Co., Ltd., he entered the Department of Electrical and Computer Engineering, YNU in 2008 and received the Research Fellowship for Young Scientists from JSPS. He received the Ph.D. degree from YNU in 2010 on chalcogenide photonic crystal waveguides and nonlinear enhancement. After studying Si photonics DQPSK receivers as a post-doctor fellow, he joined National Institute of Advanced Industrial Science and Technology and currently working on silicon photonics matrix optical switches. He is a member of JSAP. 

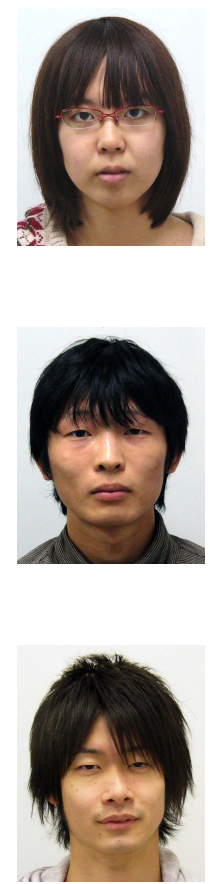

\section{Mizuki Shinkawa}

received the B. E. and M. E. degrees from the Department of Electrical and Computer Engineering, YNU in 2010 and 2012, respectively. She was studying photonic crystal slow light waveguides fabricated by CMOSprocess and its nonlinear enhancement. She is currently with Ricoh Corp., Yokohama, Japan. She is a member of JSAP.

\section{Ryo Hayakawa}

received the B. E. and M. E. degrees from the Department of Electrical and Computer Engineering, YNU in 2011 and 2013, respectively. He was studying diode-loaded photonic crystal waveguides as fast delay tuners and TPA photodiodes. He is currently with Mitsubishi Electric Corp., Hyogo, Japan. He is a member of JSAP.

\section{Keisuke Kondo}

received the B. E. degree from the Department of Electrical and Computer Engineering, YNU in 2012. He is currently working towards the M. E. degree on the dynamic tuning of photonic crystal slow light waveguides at YNU. He is a member of JSAP. He received the First Poster Award at the JSAP Annual Spring Meeting in 2013. 